\title{
Acerca del desarrollo de la estructura y la resistencia de la pasta de cemento
}

\section{RESUMEN}

La pasta de cemento, que aglomera unos con otros los granos de los áridos en el hormigón, influye decisivamente en todas sus propiedades. El Instituto de Investigaciones de la Industria del Cemento se ocupa, por lo tanto, desde hace varios años, de la estructura y de las propiedades de la pasta de cemento. En el presente trabajo se da una panorámica del estado actual de los conocimientos, obtenidos, principalmente, a través del análisis por microscopía electrónica. Los resultados explican la relación entre desarrollo de la estructura y la resistencia de la pasta de cemento y se ocupan de la influencia que en la resistencia tiene la velocidad de hidratación. Además se ensaya la posibilidad de si puede aumentarse notablemente la resistencia del mortero añadiéndole fibras muy delgadas de perlón o vidrio. Se examinan los criterios de tales mejoras.

\section{INTRODUCCION}

En el estado de partida el mortero y hormigón se componen de cemento, agua y árido. En esta mezcla el cemento forma con el agua la pasta conglomerante que rodea los granos del árido como masa plásticamente deformable. Por reacción del cemento con el agua se producen las fases hidratadas, las cuales, a medida que progresa el tiempo de reacción, aumentan entre sí y en su relación con los áridos. De este modo, el conglomerante plástico se convierte en una pasta de cemento rígida que aglutina entre sí los granos de relleno en forma de nervios delgados y sólidos.

Las propiedades mecánicas de los sólidos, por ejemplo de las piedras naturales, de los metales o de los materiales cerámicos, dependen, esencialmente, de su estructura, es decir, de la clase, tamaño, forma y disposición de sus componentes. Esto es válido también para la ıpasta de cemento. Los análisis de que se trata en este trabajo tienen por objeto acortar el conocimiento de esta relación y examinar las posibilidades para mejorar el desarrollo de la estructura y de la resistencia.

Para una hidratación total del cemento basta, teóricamente, una cantidad de agua del $38 \%$ del peso del cemento. Las cantidades de agua reales se hallan, de acuerdo con las relaciones agua/cemento de 0,4 y mayores aplicadas en la práctica, siempre por encima de este valor teórico. En consecuencia, la pasta de cemento endurecida contiene una parte de agua que no se necesita para la reacción y que, por consiguiente, existe finamente distribuida formando microporos. 
Los componentes de la estructura de la pasta endurecida, fases de hidratación y microporos, son tan pequeños que, incluso con los microscopios ópticos más potentes, no se pueden reconocer. El análisis de la estructura no se puede realizar, por lo tanto, más que con el microscopio electrónico.

\section{DESARROLLO DE LA ESTRUCTURA DE LA PASTA DE CEMENTO ENDURECIDA}

La estructura que se forma durante el endurecimiento consta, esencialmente, de cuatro fases cristalinas que contienen agua, las cuales se producen en cantidades diferentes y tienen distinta importancia en la formación de la estructura y creación de resistencia en el producto endurecido. El componente, con mucho el más importante de la estructura, es el silicato de calcio hidratado. Se produce por hidratación de los silicatos cálcicos, alita $\left(3 \mathrm{CaO} \cdot \mathrm{SiO}_{2}\right)$ y belita $\left(\beta-2 \mathrm{CaO} \cdot \mathrm{SiO}_{2}\right)$, y se compone, casi exclusivamente, de cristales fibrosos con reducido grado de ordenación estructural. La longitud de las fibras es, generalmente, del orden de $1 \mu$ y su diámetro oscila entre 0,005 y $0,1 \mu$.

La etringita $\left(3 \mathrm{CaO} \cdot \mathrm{Al}_{2} \mathrm{O}_{3} \cdot 3 \mathrm{CaSO}_{4} \cdot 32 \mathrm{H}_{2} \mathrm{O}\right)$ se forma en cristales aciculares, relativamente largos, por reacción del aluminato tricálcico $\left(3 \mathrm{CaO} \cdot \mathrm{Al}_{2} \mathrm{O}_{3}\right)$ o ferritoaluminato tetracálcico $\left(4 \mathrm{CaO} \cdot \mathrm{Al}_{2} \mathrm{O}_{3} \cdot \mathrm{Fe}_{2} \mathrm{O}_{3}\right)$ con yeso $\left(\mathrm{CaSO}_{4} \cdot 2 \mathrm{H}_{2} \mathrm{O}\right)$. Juntamente con el hidróxido cálcico es el primer producto de la hidratación que se forma después de amasarse el cemento. Por hidratación progresiva, y en presencia de hidróxido de calcio y aluminato, puede, por desprendimiento de yeso, convertirse en monosulfato $\left(3 \mathrm{CaO} \cdot \mathrm{Al}_{2} \mathrm{O}_{3} \cdot \mathrm{CaSO}_{4} \cdot\right.$ - $12 \mathrm{H}_{2} \mathrm{O}$ ). Es, por lo tanto, problemático, el que pueda emplearse esencialmente como base con estabilidad reducida para la formación de una estructura firme.

El hidróxido cálcico $\left[\mathrm{Ca}(\mathrm{OH})_{2}\right]$ se produce en la hidratación de alita y belita. El aluminato cálcico hidratado $\left(4 \mathrm{CaO} \cdot \mathrm{Al}_{2} \mathrm{O}_{3} \cdot 13 \mathrm{H}_{2} \mathrm{O}\right)$ se forma por hidratación del aluminato o del aluminoferrito, pero sólo cuando se consumió toda la proporción de yeso del cemento para la formación de la etringita. Tanto el hidróxido cálcico como el aluminato cálcico hidratado cristalizan en láminas hexagonales. Su aportación a la resistencia total de la pasta endurecida es pequeña.

Otro componente muy importante de la estructura son los poros, los cuales representan zonas débiles en la estructura total de la pasta endurecida. Como quiera que los productos de la hidratación ocupan más espacio que el cemento anhidro original, al progresar la hidratación se reduce el espacio total de los poros. En consecuencia, se altera la estructura y disminuye la resistencia. Por tal motivo, los análisis de la estructura se dirigieron preferentemente a los poros y a su alteración durante el endurecimiento. Los análisis se efectuaron, en su mayor parte, en pastas de silicato tricálcico puro $\left(3 \mathrm{CaO} \cdot \mathrm{SiO}_{2}\right)$, ya que sus productos de hidratación, silicato cálcico hidratado e hidróxido de calcio, son también de importancia decisiva para el desarrollo de la estructura de la pasta de cemento comercial endurecida. Con esta simplificación se facilitó la identificación, la mayoría de las veces muy difícil, de los componentes de la estructura.

La figura 1 muestra un poro de una pasta de silicato cálcico después de endurecida durante 3 días. Los bordes superior e inferior del poro están unidos entre sí por unas pocas fibras de silicato cálcico hidratado, o sea, que en el interior del poro el desarrollo de la estructura después de 3 días de hidratación se halla todavía en el estado inicial. Sin embargo, el interior de la fotografía está densamente lleno de silicato cálcico hidratado. En esta zona ya se ha alcanzado, por lo tanto, el estado final del desarrollo de la estructura. 


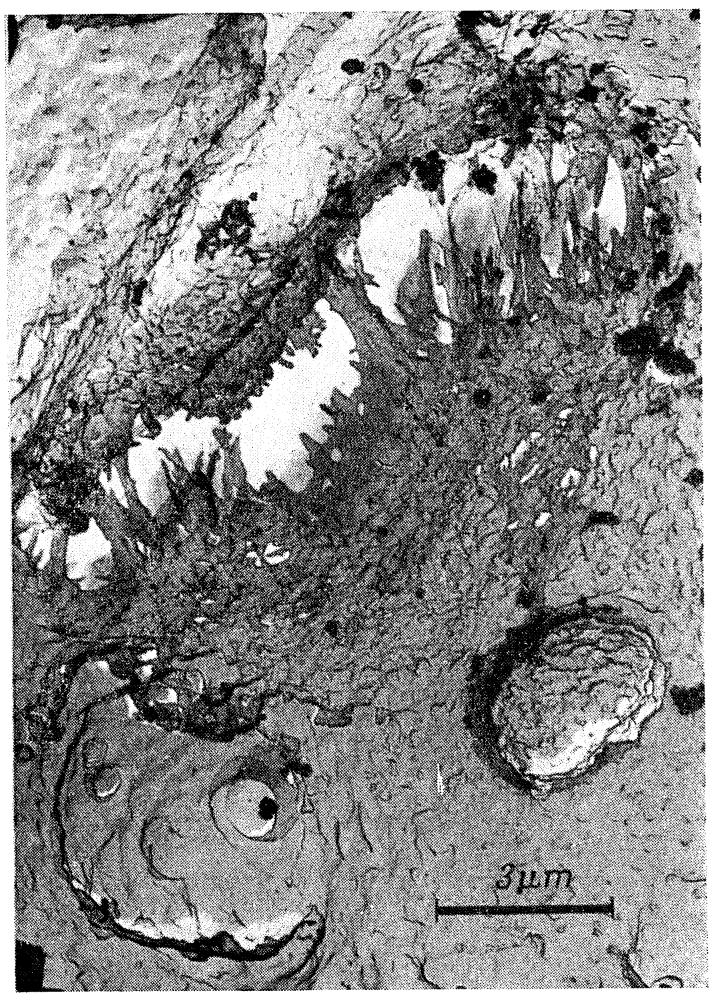

Fig. 1.-Fotografía al microscopio electrónico de la estructura de una pasta de silicato tricálcico a los 3 días; $A / C=0,44$ (réplica de carbón).

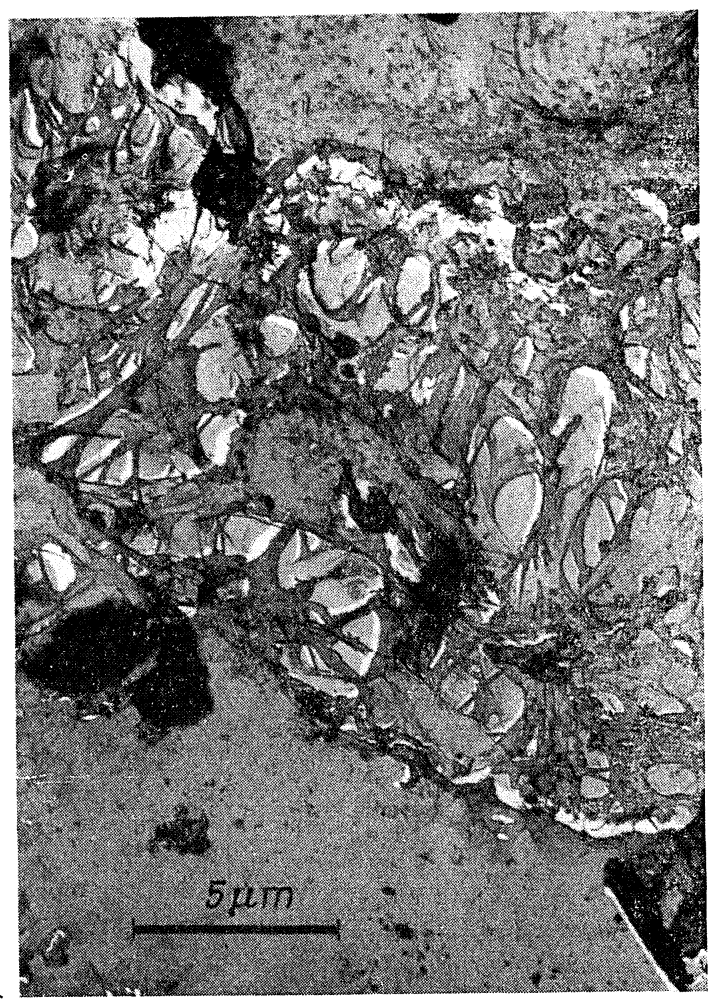

Fig. 2.-Fotografía al microscopio electrónico de la estructura de una pasta de silicato tricálcico a los 3 días; $\mathrm{A} / \mathrm{C}=\mathbf{0 , 4 4}$ (réplica de carbón).

La figura 2 muestra la zona de la estructura de un poro en principio relativamente grande, igualmente a los 3 días de edad. Cristales de silicato cálcico hidratado, en forma de fibras relativamente largas, cubren todo el espacio del poro y lo subdividen. Los poros más pequeños que se han producido en esta forma contienen fibras más cortas. De aquí se saca la conclusión de que las fibras largas de silicato cálcico hidratado se han formado en la primera fase de la hidratación, ya que sólo en este momento el espacio del poro permitía un marcado crecimiento de la longitud. Por consiguiente, después de una hidratación de 1 día ya podian reconocerse las fibras al microscopio electrónico. Al progresar el endurecimiento aumenta la subdivisión de los diversos poros y se reduce el espacio total de los mismos. Pero cuanto menores son los poros de la estructura, tanto más cortas son las fibras del silicato cálcico hidratado. De esta manera se compacta cada vez más la estructura. Esto lo pone de manifiesto la figura 3, que representa la estructura de la misma pasta de silicato tricálcico después de 90 días de endurecimiento. Se presenta aquí una estructura finamente diferenciada, la cual sólo contiene ya poros muy pequeños y que, por lo tanto, se compone predominantemente de fibras cortas de silicato cálcico hidratado. En esta estructura no faltan las fibras largas, pero en el proceso de endurecimiento se han recubierto por productos más finos de la hidratación y, por lo tanto, no son visibles en la figura.

Las figuras 1 a 3 caracterizan el desarrollo de la estructura en una pasta de silicato cálcico durante su endurecimiento. Como productos de la hidratación aparecen exclusivamente: silicato cálcico hidratado e hidróxido de calcio. Pero en la hidratación de un cemento 
portland o un cemento siderúrgico se forman además, como ya se ha indicado anteriormente, etringita, aluminato-monosulfato y aluminato tetracálcico hidratado, pero no simultáneamente, sino sucesivamente. A base de análisis radiográficos y microscópicoelectrónicos de los productos de reacción después de diversos tiempos de hidratación, se obtuvieron las relaciones reproducidas esquemáticamente en la figura 4 entre el curso de la reacción y la configuración estructural.

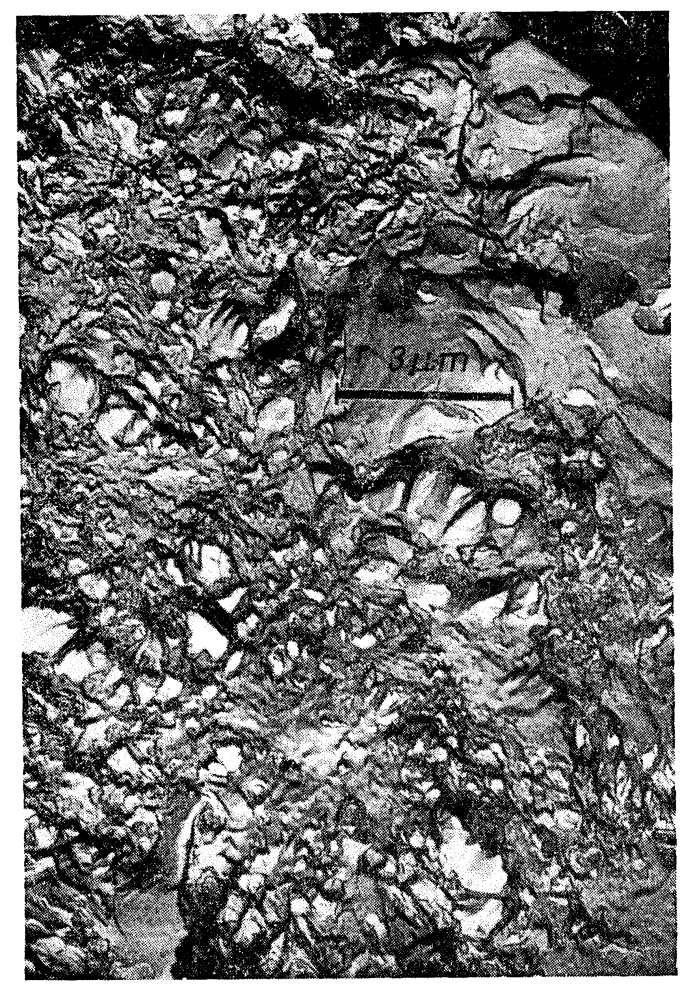

Fig. 3.-Fotografía al microscopio electrónico de la estructura de una pasta de silicato tricálcico a los 90 dias; $A / C=0,44$ (réplica de carbón).
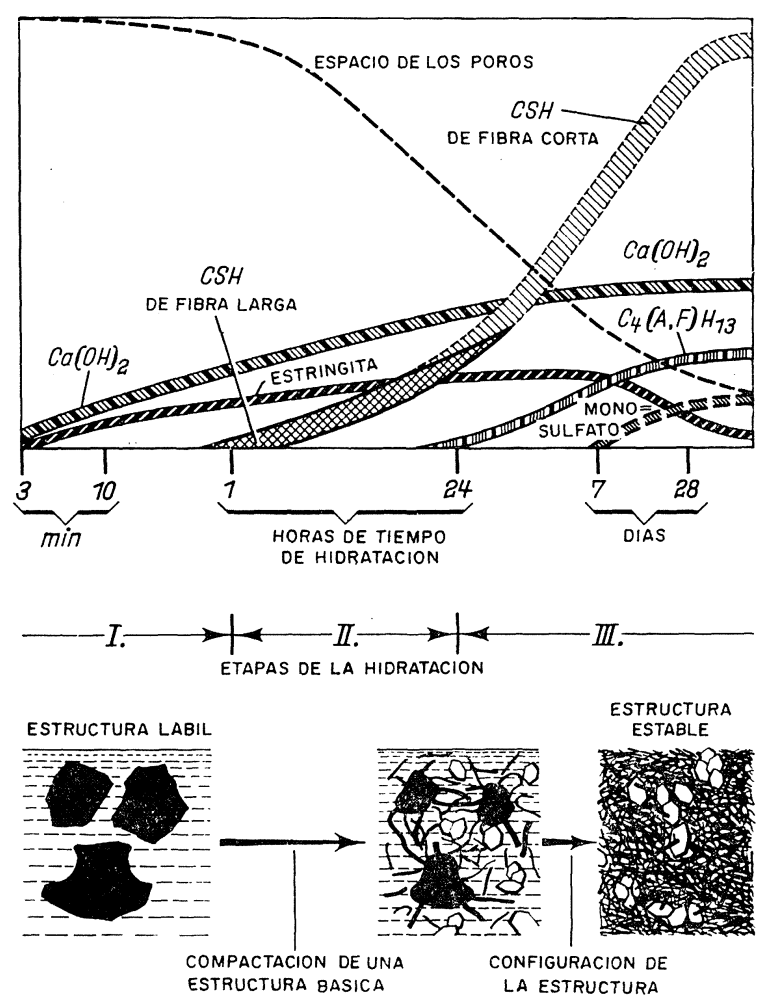

Fig. 4.-Origen de las fases de la hidratación y desarrollo de la estructura en el endurecimiento del cemento.

La primera etapa de la hidratación se caracteriza por la formación de hidróxido de calcio y etringita. Estas fases pueden comprobarse al microscopio electrónico de 3 a 10 minutos después de amasado el cemento. Todavía no existe solidificación.

La segunda etapa de la hidratación comienza, aproximadamente, 1 hora después con la formación de cristales muy finos de silicato cálcico hidratado, en primer lugar. Hasta este momento no se ha reducido esencialmente el tamaño de los granos de cemento, debido a que únicamente participó en la reacción una capa superficial delgada y las nuevas fases producidas en la hidratación son de grano muy fino.

Los espacios intermedios llenos de agua que existen entre los granos de cemento presentan, prácticamente todavía, el mismo tamaño que al comienzo de la hidratación. En consecuencia, el silicato cálcico hidratado y la etringita tienen la posibilidad de crecer en forma de fibras largas, puntear los poros $\mathrm{y}$, como se ha descrito anteriormente, subdividirles 
cada vez más. De este modo se crean amplios pilares en la segunda etapa de la hidratación con formación de una estructura básica. La resistencia final de la pasta endurecida es de esperar que será tanto más elevada cuanto mayor es la proporción de silicato cálcico hidratado de fibras largas. La segunda etapa de la hidratación, que dura hasta unas 24 horas, es, por lo tanto, de extraordinaria importancia para el desarrollo de la resistencia. Al terminar concluye también la formación de etringita, ya que después de unas 24 horas, $\mathrm{y}$ en condiciones normales de endurecimiento, se ha consumido el yeso.

En la tercera etapa de la hidratación, que se extiende hasta la hidratación total, los poros que aún existen se llenan cada vez más por los productos de la hidratación que se producen en cada caso y, como consecuencia, va aumentando la compactación de la estructura. Para ello, es condición previa de que en la pasta de cemento endurecida quede disponible el agua necesaria. En lugar de la etringita se produce en la tercera etapa de hidratación aluminato tetracálcico hidratado $\left(4 \mathrm{CaO} \cdot \mathrm{Al}_{2} \mathrm{O}_{3} \cdot 13 \mathrm{H}_{2} \mathrm{O}\right)$, en el cual se sustituye por óxido de hierro una parte de la alúmina. En esta fase de la hidratación la etringita puede transformarse en aluminato-monosulfato. Por eso es dudosa su importancia en cuanto a la resistencia final, aunque contribuye, seguramente, a la formación de la primera estructura; es decir, a la solidificación y resistencia inicial del cemento.

La limitación en el tiempo de las etapas de la hidratación, tal como se expresa en la figura 4, y sobre todo la segunda, puede afectarse si se cambian las condiciones de la hidratación. En consecuencia, como se muestra a continuación, se modifica el desarrollo de la estructura y de la resistencia. Morteros u hormigones preparados con los mismos materiales y en la misma relación de mezcla cemento/agua/árido, alcanzan resistencias diferentes si están sometidos a diferentes condiciones de endurecimiento, ya que éstas afectan, sobre todo, a las nuevas formaciones de la segunda etapa de la hidratación.

\section{INFLUENCIA DE LAS CONDICIONES DE LA HIDRATACION EN LA ESTRUCTU- RA Y EN LA RESISTENCIA DE LA PASTA DE CEMENTO ENDURECIDA}

\subsection{Endurecimiento retardado}

El cemento se hidrata tanto más lentamente cuanto menor es su finura y cuanto más baja es la temperatura de endurecimiento. La reacción se puede retardar, además, añadiendo determinadas sustancias. K. Walz y J. Bonzel, (1), informaron en 1961 que con temperaturas de hidratación bajas se retarda notablemente el endurecimiento inicial, pero que la resistencia final aumenta siempre, y advirtieron que esto podría depender de la configuración de las nuevas formaciones que con temperatura más baja se producen más lentamente. Resultados prácticamente iguales obtuvieron O. P. Mtschedlow-Petrossian y W. L. Tschernjawski, (2), enfriando durante 24 horas (hasta $-20^{\circ} \mathrm{C}$ ) muestras de mortero y endureciéndolas a continuación a temperatura normal. Estos resultados pueden explicarse como siguen: con temperatura baja es más lenta la reacción del cemento con ę agua de amasado; por unidad de tiempo se producen menos productos de la hidratación y como consecuencia el cemento se endurece más lentamente. El retraso de la reacción produce, además, que las etapas de la hidratación se prolonguen más de acuerdo con la figura 4. En consecuencia, sobre todo en la segunda etapa de la hidratación, puede formarse una estructura básica con una mayor proporción de silicato cálcico hidratado de fibra larga, que, por amplios refuerzos y con un número respectivamente mayor de puntos de contacto, originan una mayor resistencia final. De la influencia de la temperatura de endurecimiento en la morfología de los silicatos cálcicos hidratados ya se informó en otra comunicación, (3). 
Análogos cambios en la estructura y en el desarrollo de la resistencia los originan aditivos químicos que retrasan el endurecimiento del cemento. La figura 5 reproduce la estructura de una pasta de silicato tricálcico a los 7 días, cuyo endurecimiento se retrasó con $1 \%$ de óxido de zinc. En estas condicio-

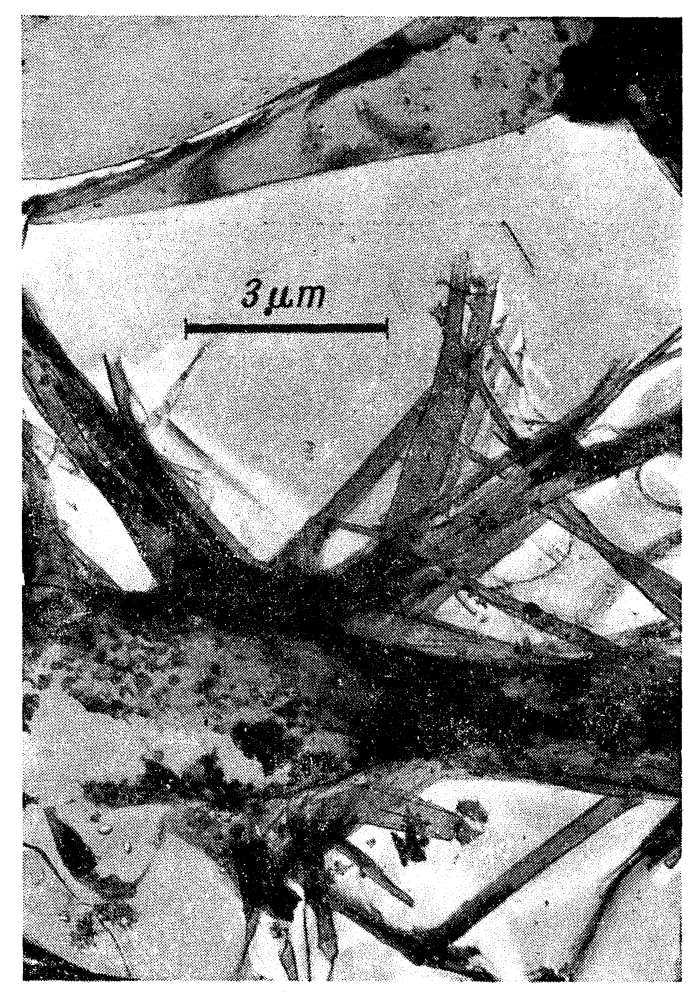

Fig. 5.-Fotografía al microscopio electrónico de la estructura de una pasta de silicato tricálcico a 7 días, cuyo endurecimiento se retrasó por la adición de $1 \%$ de óxido de zinc; $A / C=0,44$ (réplica de carbón). nes, parte de las fibras del silicato cálcico hidratado alcanzaron longitudes de hasta 10 $\mu$; en algunos casos se midieron, incluso, hasta $30 \mu$. De acuerdo con la estructura, la resistencia final es considerablemente mayor que con un endurecimiento normal. Ensayos de W. Lieber, (4), dieron por resultado que la adición de óxido de zinc desciende fuertemente la resistencia inicial, pero la resistencia final aumenta del orden del $20 \%$. Resultados parecidos han expuesto $\mathrm{K}$. Walz y $\mathrm{H}$. Mathieu, (5), quienes analizaron la influencia de diversos retardantes en el desarrollo de la resistencia de los cementos, comprobando siempre que se obtiene una resistencia final mayor cuando se retrasó el endurecimiento inicial.

La causa de la influencia en el retraso aún no ha sido aclarada, pero puede suponerse que los aditivos bloquean primeramente la superficie de los granos de cemento $y$, en consecuencia, retardan las reacciones de hidratación.

(continuará)

\section{BIBLIOGRAFIA}

(1) Walz, K., und J. Bonzel: Festigkeitsentwicklung verschiedener Zemente bei niederer Temperatur. beton 11 (1961) H. 1, S. 35/48; ebenso Betontechnische Berichte 1961, Beton-Verlag, Düsseldorf 1962, S. 9/46.

(2) Mtschedlow-Petrossian, O. P., und W. L. Tschernjawski: Einfluß niedriger Temperaturen auf den Hydratationsprozeß von Portlandzementen. Silikattechnik 18 (1967) H. 3, S. 72/76.

(3) Richartz, W., und F. W. Locher: Ein Beitrag zur Morphologie und Wasserbindung von Calciumsilicathydraten und zum Gefüge des Zementsteins. Zement-Kalk-Gips 18 (1965) H: 9, S. 449/459.

(4) Lieber, W.: Einfluß von Zinkoxyd auf das Erstarren und Erhärten von Portlandzementen. Zement-Kalk-Gips 20 (1967) H. 3, S. 91/95.

(5) Walz, K., und H. Mathieu: Einfluß der Zusatzmenge von Betonverflüssigern auf die Festigkeitsentwicklung. beton 11 (1961) H. 9, S. 619/624, und H. 10, 691/696; ebenso Betontechnische Berichte 1961, Beton-Verlag, Düsseldorf 1962, S. 241/266. 